\title{
INDEX
}

Note: an 'n' after a page number denotes a footnote.

Aboriginal and Torres Strait Islander Commission (ATSIC) 170-1

Aboriginal and Torres Strait Islander Social Justice Commission 165

Aboriginal Australians see Indigenous Australians

Aboriginal Medical Services 183, 187-8, 195

Aboriginal Tent Embassy 175

abuse $7,43,56,79,159,201,209-10$ causes $14,65,81$

social structure 13, 34, 63, 203

state $34,86,121,126,149,162-3$, 201, 202-3

cultural specificity $77-8$

defined 4

economic 81, 144, 202

legal mechanisms 107, 205-6

patterns of 13, 123, 128, 209

recognition of $15,126,206-7$

as basis of human rights $79-80$

responses to $11,15,95,122-3,159$,

211

difficulties of 8,9

international 12, 202-3

long-term dialogue 90-1

role of state $86,101,107,121-3,149$, 159, 201, 205-6

systemic 202

and Aboriginal health 162-3

China 121, 126

East Timor 130, 143, 144

see also suffering

activism 34, 79

Aditjondro, George 148

Alfonsin, Raoul 159

Amnesty International 164

anthropology 59-60, 78-9
Apodeti 133, 136, 139, 140

Argentina 159

'Asian Way' debate 6,98

abuse $77,79-80,81$

colonialism 78-9

community $80-1,201$

cultural arguments 78-9

East-West dichotomies 79-81

economic verses civil rights 76,81

explained 76,78

human rights recognition $79-80,81$

individualism $76,80-1$

origins of $76-7$

perceptions of $76,77,79$

political issues $77,78,79$

universalism-relativism debate 79,80 , 81,82

Western assumptions of superiority $77-8,81$

Asia Watch 45

ATSIC (Aboriginal and Torres Strait Islander Commission) 170-1

Australia 167-8

and East Timor 134, 142, 152

support for integration 136, 142

see also Indigenous Australian health

authoritarianism $76,104-5$

\section{BAKIN 138-9}

Beijing Spring 95, 97, 102-3, 110, 118

criticisms of students' motives 111-12,

113, 114, 120

by Liu Xiaobo 116, 120

demands 94, 100, 101, 113, 114

participation and accountability 112 ,

115,117

democracy, understanding of 111,113 ,

117-19, 120 
ambivalent attitude of intellectuals

117-19

hunger strikes 109, 115, 116, 120

mythologising of students 110-12,

116-17

dangers 111-12

official response 102-3, 110-11

patriotic nature of student movement 115,117

role of students 110-21

and martyrdom 99, 111, 116-17

as 'revolutionary' 116-17

role of workers 110

scope $94,110,127 \mathrm{n} 1$

support for $94,101,121$

Western interpretation 111, 113

Belo, Monsignor 145

Bringing Them Home Report 165

see also Indigenous Australian disadvantage

Brown, Chris 62, 63-4, 65-6

Caetano dictatorship 133

capitalism 26, 29, 98, 100

case studies 17-18, 90-2

see also East Timor; Indigenous Australian health; Tiananmen Square massacre

Catholic Church 23

and East Timor 131, 133, 134, 148, 156

see also Christendom; religious traditions

CERD (Convention on the Elimination of Racial Discrimination) 167

Chen Xianda 98, 99

childhood 29-30

China 98, 119

China Democratic Party 97

communism 104, 106, 112-14

Communist Party 97, 103-8

need for reform 123, 124

Confucianism 76, 78, 114-15, 116, 126-7

Cultural Revolution 101, 108

democracy 111, 113, 117-19, 120

Democracy Movement 94, 108-9, 119, 123

Democracy Wall 115, 119 dialogue 114, 115-16

economic uncertainty 105, 106,

112-13, 115

erosion of social values $106,108,112$

income disparity 105-6, 112-13

international relations $96,97-8$

leadership 102-3, 105, 106, 107, 116, 197-8

modernisation 96, 103-4, 106, 108

corruption 106, 108

economic 95, 96, 105, 115

employment 112-13

impact on students 112, 115

and leadership 104-5, 107

and rights 106, 108-9, 124

standing of intellectuals 112-13, 115

urban reforms 112-13

official attitude to human rights $97-8$, 99

participation in local politics 119-20

People's Liberation Army 110, 117

crisis of loyalty 109-10

role in Tiananmen Square massacre

94, 101

reform 107, 114-15, 126

expectations of 107,112

and intellectuals 107, 114-15.

117-19

models for $104-5$

response to sanctions $96-7$

rights 93, 106, 123-4, 125, 126

citizens 98-9, 107

to subsistence 98,108

social welfare $104,105,106$

state, role of 98, 102-10, 115

students $114,120,121$

desire for dialogue 115-16

economic change 112, 114

workers $110,111,114,115,119,120$, $127 \mathrm{n} 4$

see also Tiananmen Square massacre

Christendom 24-5, 58

see also Catholic Church; religious traditions

church missions 171-2, 181

citizenship 69

construction of $173,188,191$ 
and Indigenous Australians 173-4, 180, 182-3, 190-2, 193, 196, 208

citizens rights $86,91,93,98-9,101,125$. 199

civil rights 96, 107

prioritised over economic $34,76,80,81$

civil society $26,29,33,201$

Cold War 50, 98

and East Timor 129, 136-7

and Tiananmen Square massacre 93, 96 , 97

colonialism 34-5, 59-60, 82, 202

impact on culture 78-9

and indigenous peoples $167,186-7$

universalism-relativism debate 55, 59, 60

colonisation 9, 195

and 'Asian Way' debate 76-7

East Timor 130-3

Indonesia 129-30, 132

see also decolonisation

Commission of Investigation into Human

Rights Violations (Indonesia) 154

communism

China $104,106,112-14$

and East Timor 136-7, 138

see also Marxism

Communist Party (China) 97, 104, 108

leadership 105, 106

need for reform 123, 124

role of 103, 107

communitarianism see 'Asian Way' debate community 18, 67, 80-1, 206, 207

'Asian Way' debate 80-1, 201

in Indigenous Australia 170, 187, 208

political $130,188,191$

rebuilding after abuse $21-2,91,128$, 209

and rights $87,206-7$

state as $63-4,80,88 \mathrm{n} 5,208$

Confucianism $76,78,114-15,116,126-7$

contractarianism 20, 22, 25, 35-6, 47-8, 86

and utilitarianism 23-4

Convention on the Elimination of Racial Discrimination (CERD) 167

Council for Aboriginal Reconciliation 165 critical theory $18 \mathrm{n} 4,50,67,68$

cultural imperialism 77,79

Cultural Revolution 101, 108

culture $65,69,74,78,90-1$

'Asian Way' debate 77, 78, 80

impact of colonialism 78-9, 131

'Dare to Die' corps 111

decolonisation 60, 179

and 'Asian Way' debate 76-7

East Timor 130, 132, 133, 161

and terra nullius 195-6

see also colonisation

definitions 9, 15, 19, 21, 35, 83, 206

democracy 91, 155

see also China, democracy; liberal democracy

democratic capitalism 101-2

Deng Xiaoping 105, 109, 127n 3

and leadership 104, 107, 110-11

development, and rights 81, 98, 149

dialogue 56, 71, 82-6, 195

and China 114, 115-16

and East Timor 160

explained $82,83,84$

Indigenous Australian disadvantage 163, 193, 194, 195, 196

limitations of 71,85

Linklater, Andrew 67, 69, 70-1, 74, 82

and listening 84,85

'message' approach 82, 202

need for on-going 90-1, 211

and rights $82,85-6,90$

Tiananmen Square massacre 93, 109, $110,114,115-16,121$

and understanding $84-5$

difference 59-60, 65, 67, 70, 100, 175

assumptions of Western superiority 65-6

denial of Indigenous Australian 190-1, 194

engaging with $13-14,74$

exclusion based on 29-30, 31, 32

oppositions $11-15,20,57,79,80$

and rights $11-12$

as site of abuse 14 
differentiation $6,58,59$

diversity see difference

Dodson, Mick 188-9, 190, 192-3, 194

'domestic' human rights 10-11

domestic violence 29, 35

Donnelly, John 5, 6

East Timor $80-1,128,130,131,135-6$, $150,151-2,160-1$

and abuse $129,130,143-4,145,148$, 149,150

following vote $153-4,158,159$

Apodeti 133, 136, 139, 140

and Australia 134, 136, 142, 152

Catholic Church, influence 133, 134, $145,148,156$

and Catholicism 131, 134

as rejection of Indonesian identity 131,148

colonisation 130-3

abuse during Portuguese rule 131, 133

coup 139-40

decolonisation 130, 132, 133

right to 161

UN support 129-30

differences from and similarities with Indonesia 130-1

economic development 81, 144, 146, 149

cost to Indonesia $145,148,150-1$

and Indonesian military 145-6,

152-3

legacy 145-6, 149-50

under Portuguese 131, 133, 136

Fretilin 134, 140, 156

emergence of 133, 134

Marxist elements 134, 136-7

supported by Portuguese 134, 135

and UDT $135,138,139-40$

history 128, 129, 130-43

human rights situation $143-50$

IMF 156, 157

integration 139, 140-1, 142, 143

economic impacts 144

Indonesian belief in desire for 147 , 150-1, 153 influence of internal Indonesian

politics $137-8$

other models $157-8$

reversed 128

international fears of communism 134, $136,137-8$

invasion 140, 141, 146

impact on Timorese 144-5, 148, 149, $150,158-9$

international acquiescence 129-30, 140, 161

international responses 136,140 ,

142-3

scope 143-4

peace building 91, 155, 159-60

threats to $156-7,159$

and Portugal 130, 131, 133, 134-5

response to coup 139-40

resistance 133-40, 143-4

divisions within $135,148-9,157-8$

Xanana Gusmao 146, 152, 158-9

Santa Cruz massacre 129, 146-8

Indonesian defence of 147,148

Indonesian investigation $147-8$

responses 147,148

Second World War 132

self-determination 133, 142, 152

ambiguity of term 160

economic factors 135,136

emergence of political parties 133-4

Indonesian interpretation 138-9

international assistance 155,156

international rhetoric 139, 140-2

lack of confidence in 135, 136

as rhetoric of Portuguese governments

$133,134-5$

terror campaign 153

and UN 128-30, 153

violence following vote $153-4$

state 155-6

conflict management 155-6, 158,

159

construction of $130,135,155,157$,

160

nation building 91, 128

and participation 141, 143, 156, 157,

158, 208 
reconciliation 159-60, 208, 209

transition 157

fear of cost 133, 136, 139, 152

strategic concerns $136-7$

UDT (Uniao Democratica de Timor)

$133-4,135,138,139-40$

UNTAET 128-9, 154-5, 160

as de facto government 128,155 , 157,158

US foreign policy $129,136-7,140$

economic development 81, 104, 112, 136, 172, 196n 3

see also East Timor, economic

development

economic liberalisation 105, 106, 210

in China $104,106,112,114,115$

and peace building $156-7$

economic rights $29,32,34,47,81,164$, 199, 210

civil prioritised $34,76,80,81,108-9$

overshadowed 76

empathy, as basis for morality $62-3,65$, 66,67

Enlightenment, the 24, 26-7

ethics 23, 40, 51, 83

and human rights $16,17,18 \mathrm{n} 1$

and power $41-2$

religious traditions $74-5$

universalism-relativism debate 55-6

'ethnic cleansing' 59

ethnocentrism $42,47,59,65-7,75,77-8$, $126-7$

EU (European Union) 68-9, 130

European Union see EU

everyman see universal man

exclusion 14, 21, 29, 67, 73

based on difference 29-30, 31, 32

and human rights $10-11$

family $28-9,30$

first-generation rights $32-3,34$

foreign policy 41-2, 129, 136-7, 140

Foucault, Michel 41, 57

Fretilin see East Timor, Fretilin

Gadjah Mada Research Centre report 146 , 150 gender $28-9,30,31$

globalisation 47, 167

global norms see international norms

Goa 140, 143

Gomes, Costa 133, 135

Gongzilian (Beijing Workers' Autonomous Union) 120

Gusmao, Xanana 146, 152, 158-9

Habermas, Jurgen 67, 71

harm see suffering

health see Indigenous Australian health

Hobbes, Thomas 25, 26, 35, 37, 98

Holland see Indonesia

homogeneity 11-12

see also difference

House of Representatives Standing Committees on Aboriginal Affairs (Australia) see HRSCAA

HREOC (Human Rights and Equal Opportunities Commission) 172, 191-2

HRSCAA (House of Representatives Standing Committees on Aboriginal Affairs) 184,185

humanness, notions of $15,18,21,22$

Human Rights and Equal Opportunities Commission (Australia) see HREOC

Human Rights Commission (Australia) 165

hunger strikes 109, 115, 116, 120

Hu Yaobang 104-5, 107

ICCPR (International Covenant on Civil and Political Rights) 97

idealism 50, 51-2

definitions of 52

and realism 20, 37, 50-3, 125, 160-1

ILO (International Labour Organisation) 97

IMF (International Monetary Fund) 155, $156-7$

Immigration Restriction Act (Australia) 180

inclusiveness 31-2, 67, 69

Indigenous Australian disadvantage 91, 162, 163, 201

assimilation policy $174,181,182$, 190-1, 193 
assumptions regarding $165,174,181$, 184

culturally based $165,167,171,180$

social Darwinism 179-80

causes of 162-3, 171, 181

'Aboriginal problem' 176, 185-6

settler attitudes $167,172,178,179$, 182

see also under Indigenous Australian health

child removal $167,179,182$

Bringing Them Home Report 165

church missions 171-2, 181

colonialism 186-7

deaths in custody 165,166

denial of difference 190-1, 194

dependence 171-2, 181, 188-9, 191

dialogue, need for 163, 193, 194, 195, 196

dispossession 171, 172-3, 175, 178-9, $180,181,191$

economic control 171-2, 178-9, 181, 188

education 170

efforts to redress $162,165,171,181-2$, 191-2

see also Indigenous Australian health, policy

equity issues $174,190,194$

extent of $166,167,168-77,186$

housing $170-1,181,185$

impacts of abuse 171-2, 173-4

incarceration 166, 181

infrastructure, lack of $170-1,172$

institutionalisation $181-2,188$

international response $164-5,167,182$

land, loss of see Indigenous Australians, land rights

marginalisation $164,174,175,177-82$, $181,183,194$

national concern $163,165-6,167$

poverty $162,170-1,181,184$

public services $170-1$

representations of Indigenous people 179-80, 181

responsibility for $166,178-9$

unemployment 170 violence $162,163,169-70$

welfare $172-3,174,175,196 \mathrm{n} 3$

welfarism 173, 175, 196n1

White Australia policy 180

Indigenous Australian health 91, 162,

$166,187,192,193,208$

causes of death 162,164,169, 184

infant mortality 162, 168, 181, 186

social conditions 169,170

substance abuse $162,169-70$

suicide 162

violence $162,169-70$

causes of ill-health 181,187

lack of infrastructure 170-1, 183, 184-5

malnutrition $169,170,181,185$

poverty $162,170-1,181,184$

and citizenship 174, 208

comparison with settler health 168-9

criticism by international bodies 163-4, 182

government response to 165

cultural awareness $176,183,184,185$, $186-7,188,196-7 \mathrm{n} 4$

cultural beliefs 187

current conditions $167,168-77$

statistics $168,169,171,186$

environmental health 162, 170, 184

government response see under policy below

and human rights $164,166,174,194$, 204

international criticism $163-5,182$

lack of improvement 169, 186

life expectancy 168

mental illness 170

national attention 168,176

participation $163,183-4,188$

consultation $177,186,196-7 \mathrm{n} 4$

and mutuality 192, 193

policy $162,165,182-8,191,201$

Aboriginal Medical Services 183 ,

$187-8,195$

consultation 188

control of Indigenous lives 163, 182, 183

funding $176-7,183,184,185,189$ 
impact of 183, 184, 185-6, 187

infrastructure a state responsibility 184-5

lack of accountability 184-5, 186, 189

lack of cooperation 183, 184-5, 186, $187-8$

liaison officers 181-2

national Aboriginal health organisation $187-8$

National Aboriginal Health Strategy 185

national database 184

national plans $183-4$

1967 referendum $182-3$

self-management $175,184,188,197 \mathrm{n} 8$

substance abuse $162,166,169-70$

Indigenous Australians 167, 173-5, 179

activism 174, 182-3, 192

apology by government 165,195

citizenship 173-4, 190, 191-2, 193. 196

assimilation 190, 191

attainment of $167,180,182-3$

and health 174, 208

1967 Referendum 167, 182-3

community 192

importance of 170, 187, 208

history, need for acknowledgement 194-5

land rights 162, 165, 178-9, 190, 192, 194, 210-11

and health 162, 182, 192

native title $178,192,193$

terra nullius 177, 180, 191, 195-6

Wik decision 166, 178

see also Mabo decision

multiculturalism, rhetoric of 174

participation in political life 91, 164,

172, 175, 195, 207

barriers to $188,191-2,193$

reconciliation 165, 174, 193, 208

rights 91, 163, 188, 192, 193, 194

citizenship 173, 174

and health 164, 166, 174, 204

welfare $172-3,175,177,196 \mathrm{n} 3$

self-determination $174,175-6,188-9$, 191, 192-4 economic 196n3

Tent Embassy 175

self-government 175-6

treaty 175,195

indigenous peoples $7,31,167$

individualism 6, 27, 61, 99, 211

and human rights 199, 200, 201

see also universalism-relativism debate

Indonesia 79, 137-8, 139, 151-2, 154

anti-communism 137-8

belief in 'saving' East Timor 145, 147, 152

colonisation 129-30, 132

coup of 1965137

criticism of military 147-8, 151

desire for consolidation 132, 136, 137, 138

differences from and similarities with East Timor 130-1

fear of fragility of state 132, 137, 138, 151

human rights situation $79-80$

independence movement 132, 137

international relations $139,142-3$

response to condemnation 143, 149

leadership struggles 138-9, 151-2

security as priority $144-5,151$

sense of nationhood 132, 138

Suharto $79,81,137,139,151-2$

see also East Timor

Indonesian Commission of Investigation report 154

injury see suffering

interdependency theory 48-9

International Covenant on Civil and Political Rights (ICCPR) 97

international diplomacy 48, 49

International Labour Organisation (ILO) 97

International Monetary Fund (IMF) 155, $156-7$

international norms 43-5, 46, 47, 49, 63, 167, 202-3

international relations $19,20,35-6$, $37-54$

and contractarianism 35

homogeneity and difference 11,12

and human rights $3,10-12,43$ 
and power $39-40$

and realism 37,38

Irian Jaya 140

\section{Kantian perspectives 51,67} see also Linklater, Andrew

labour organisations 94

land rights see Indigenous Australians, land rights

learning 45-6, 48-9, 84

liberal democracy 13, 69 construction of citizenship 188, 191 and East Timor 129, 155 and human rights $5,6,8,12,19,83$ liberalism 43, 44-6, 47, 49, 56, 200

'Asian Way' debate 76 construction of rights 203-4 and human rights 43, 49, 200 and realism 43, 44, 50 state-centred nature of $46-7$ and universality 46,61 and utilitarianism $43,44,49$ liberty, definition of 33

Linklater, Andrew 67-74 critique of state $73-4$ emphasis on dialogue $67,69,70-1,74$, 82

and universalism 69-70, 71

Li Peng 116

listening $7,16-17,21,84,85$

Liu Xiaobo 116, 120, 127

Locke, John 25, 31, 35, 179

contractarianism 20, 22-3, 35, 37, 178

derivation of rights $5,6,17,28-9$

see also social contract

Lockean framework and abuse of rights

$$
111-12,121-3
$$

Mabo decision 165, 166, 167, 177-9, 180, 190, 194

implications of $177-8$

international law 196, 197n9

native title $178,192,193$

and terra nullius $177,180,191,195-6$

see also Indigenous Australians, land rights
Malik, Adam 138, 140

'man' see universal man

market economy see economic liberalisation

Marsinah 79, 80

Marxism 31, 51, 67, 112, 206

and Chinese reform 104-5

influence on Fretilin 134, 136-7

and rights 50, 99

middle classes 26, 31

migration 63-4, 68-9

modernisation 35, 78, 96

see also China, modernisation

modern state 36

emergence of $6,8,18,27$

see also Westphalian system

Mohamad, Goenawan 79-80

moral obligation see Rorty, Richard

moral superiority, assumptions of $62,65-7$

Murtopo, General 135

NACCHO (National Aboriginal Community Centre Health Organisation) 187-8, 195

Nandy, Ashis 75, 76, 79, 82

National Aboriginal Community Centre Health Organisation see NACCHO

natural law 22-3, 24, 53n1

negative rights $32-3,34$

neorealism $37-8$

NGOs (non-governmental organisations) 45,153

non-interference, principle of see Westphalian system

origins of rights $19,22-3,25-6,28-9,43$, 49

pain see suffering

Parekh, Bhikhu 62, 74

Parliamentary Sub-Committee on Human Rights (Australia) 170

Pearson, Noel 166, 190

People's Liberation Army see China, People's Liberation Army

Politburo Standing Committee (China) 110

political rights $34,96,199$

Portugal see East Timor 
positive rights 33,164

postmodernism 72

poverty 81,158

cause of ill-health $162,170-1,181,184$

Indigenous Australian disadvantage 162, $170-1,181,184$

power $29,45,54 \mathrm{n} 5$

and ethics $41-2$

international relations $39-40$

and realism $39-41,42$

rise of secular $6,24-5,28$

and social contract $29-30$

'soft' 45-6

practice of human rights $4-5,6-7,47$,

204, 205, 209, 210

and 'community' 206-7, 209

confrontation in 208-9

countering abuse $79-80,90,205-6$

inclusivity $207-8$

use of threats 202, 208

pragmatism 65,96

promotion of rights 4, 90-1, 198, 199, 200, 202

as agent of change 12,13

international 43, 55-6, 76, 198

language of 19-20, 199

and modern state $8,10,198-9,202-3$

progress in 35,46

relationship between state and individual 199, 202-3

shaping debate 199-200

see also 'Asian Way' debate

Racial Discrimination Act (Australia) 167, 191-2

radical fragmentation, principle of 61

Ramos-Horta, Jose 134, 138

rationality, rejection of $62,64,65$

RCIADIC (Royal Commission into

Aboriginal Deaths in Custody) 165 , 175-6, 182, 186

realism $37-8,39,41,42-5,54 n 6$

conflicts within $41-2$

definitions 38-9

and ethics 40, 41-2

'hard' 37, 38, 39

and idealism $50-1,52-3$ and liberalism 43, 44, 50

origins of $37,38,39$

and power $39-41,42$

rebuilding community $21-2,91,128,155$, 159-60

relativism 20, 52, 55, 57, 62, 206

and Brown 63-4

equated with 'East' 79, 80, 81

and Rorty $66-7$

see also universalism-relativism debate

religious traditions $39,53 \mathrm{n} 1,74,75$

Christendom 24-5, 58

church missions 172, 181

origins of human rights $6,24,25,27$

and violence $75,87 \mathrm{n} 2,148,151$

see also Catholic Church

rights $41,47,78,86,87,98,164,200$

civil 32, 96, 107

prioritised over economic 34, 76, 80, $81,108-9$

complexity 122, 198, 203

concepts 3, 4-6, 8, 9-10, 13, 35

construction $47,55,200-1,203-4$

critiques of $34,42,50,55$

definition of $86-7$

derivation of $5,6-7,28,199$

Lockean social contract $17,28-9,32$

to development 81

and dialogue 85-6

economic 29, 32, 34, 47, 81, 164, 199, 210

first-generation negative rights $32-3$, 34

political 32, 34, 96, 199

second-generation positive rights 33 , 164

universality 36,61

rights promotion 87,123

'message' approach 47, 82

Rorty, Richard 56, 62, 68

assumptions of moral superiority $65-7$

empathy $62-3,65,66,67$

rationality $62,64,65,72$

transcultural morality $64,65,71$

universalism-relativism debate $62-7$

Royal Commission into Aboriginal Deaths in Custody (Australia) see RCIADIC 
sanctions $96-7$

second-generation rights 33,164

secular state, rise of $6,24-5,28$

self, construction of $47-8$

self-determination 98

see also East Timor, self-determination

self-interest $45,61,65$

self-reflection 47,82

slavery 30

social and cultural rights 199

see also Indigenous Australian health

social contract $5,25,37$

description of state 33-4

exclusivity of 31-2

explained $26-7$

origins 24

theories 24-36

see also Hobbes, Thomas; Locke, John

social duties versus rights 25

socialism 99

see also communism; Marxism

sovereignty $83,98,99$

Soviet bloc 99, 100

Standing Committee of the National People's Congress (China) 109-10

Stanner, W. E. H. $167,177-82$

state $59,69,73,93,103,108-9,174$,

201-2

as cause of abuse $34,86,121,126$, $149,162-3,201,202-3$

central to liberalism $46-7$

as community $63-4,80,88 \mathrm{n} 5,208$

critique of $73-4$

cultural influences 76,78

development of $55,58-9$

modern $6,8,18,27,36,60-1,198-9$

secular $6,24-5,28$

impact of human rights $11,76,106$, 198-9, 200-1

liberal-democratic, and human rights 5 , $6,8,12,19,83$

relationship with individual 102,199 , 202-3

China democracy movement 93, 94-5, 101, 102, 125-6

and social contract $33-4$

sovereignty $54 \mathrm{n} 5,68-9,98,202$ universality 55, 59, 202

Westphalian system 8,11

see also China state, role of; East Timor

subsistence 108, 210

as right 32,98

suffering $8-9,10,47,75,85,162$

defined 4,5

recognition of 9, 23, 199

relationship to abuse 204-5

responses to $49,57,67,83,85,205$

roots of 48

see also abuse

Suharto $79,81,137,139,151-2$

terra nullius see Mabo decision

Tiananmen Papers, The 93-4

Tiananmen Square massacre 9, 93-127

aftermath $94,95,97,101,121,123-4$

causes $121,123,124$

citizens rights above 'human' rights 91,

93, 98-9, 101, 124-5

individualism 99

inevitability of 102,110

international relations $96,97-8$

leadership, state 94, 95, 108-9, 110, $120-1,122-3$

and dialogue 93, 110, 121

Liu Xiaobo 116, 120, 127

oversimplification of 121-3, 126

pragmatism-realism debate 96,97

representations 95, 99

in China 99, 101

as 'man' versus 'state' 94-5, 101, 102

in West 99, 100, 101, 102

responses $95,96,102,121-7$

demonisation of Chinese regime

99-100, 101, 122

impact of Lockean model 91, 93, 95, 101

sanctions $96-7$

Western mythologising 95, 101, 111,

121-2, 125-6

rights debate within China 96, 122,

123-4

supremacy of citizen's rights $98-9$, 101 
significance

in China 93, 95

in West 93, 94-5, 96, 99, 101

state, concept of 93, 201

students 95,122

as martyrs $99,100,101$

support for 109-10

workers 111

Zhang Liang 93-4

see also Beijing Spring; China

Tibet 95

Torres Strait Islander people see Indigenous Australians

torture 14-15

see also abuse; suffering

trade sanctions $96-7$

truth $16,42,71-2,75,83,201-2$

and idealism 50, 51, 52

and 'message' approach to rights promotion $47,82,90-1$

Truth and Reconciliation Commission 15

UDT (Uniao Democratica de Timor) 133-4, $135,138,139-40$

UN (United Nations) 20, 24, 44-5, 46, 156

and East Timor 129-30, 132, 153

see also UNTAET

UN Committee Against Torture 165

UN Committee on the Elimination of Racial Discrimination 165

UN Covenant on Economic, Social and Cultural Rights 164

UNHRC 97, 165

UN Human Rights Commission see UNHRC uniformity $58-9$

United Nations see UN

United States 97, 164

Universal Declaration of Human Rights 24, $46,57,167$

Universal Human Rights Covenant 46

universalism 56-7, 59, 62, 70, 74, 199

alternative $75-6$

critiques of 56,57

defined 83

equated with 'West' 79, 80, 81

and human rights 200-1 limit of concept 55, 57

see also Brown, Chris; Linklater, Andrew;

Rorty, Richard

universalism-relativism debate 20, 57, 60-2, 67, 74, 83, 202

deficiencies of $60-1,67,74$

development of modern state 60-1

and ethics 55-6

and rights $60-1,67,200$

and sovereignty 58,83

see also 'Asian Way' debate; Brown,

Chris; Linklater, Andrew; Rorty,

Richard

universalist-utilitarian approach 200-2

universality $6,8,21,30-1,32,47-8,52$

defined 8,68

and idealism 50

of realism 39

reconceptualised $67-8$

and rights $8,9,12,33,36,46,61$, $86-7,199,200-1$

see also Linklater, Andrew

universal man $27-8,30,31,58$

construction of 188,191

and Enlightenment 24-5

versus the state $94-5,101$

UNTAET 128-9, 154-5, 160

as de facto government $128,155,157$, 158

UN Transitional Authority for East Timor see UNTAET

utilitarianism 23-4, 49, 200

utopianism see idealism

Vienna Conference on Human Rights 77, 98

violence see abuse; suffering

welfare rights see second-generation rights

Western concepts of rights $34-5,47,59$, $79,126-7$

Westphalia, Peace of 24

Westphalian system 16, 24, 43-4, 67, 69

concept of the state 8,11

definition 18n2, 24

universalism 58, 201-2

Whitlam, Gough 136, 142 
Index

Wik decision 166, 178

see also Indigenous Australians, land rights; Mabo decision

Wittgenstein, Ludwig 8, 72, 73

women $7,30,34$

workers 7,79-80, 180, 210

and democracy movement 110, 111, $114,115,119,120,127 \mathrm{n} 4$
World Bank 96, 155, 156-7

World Council of Churches 164

Wu'erkaixi 115-16, 117

Yang Shangkun 109

Zhang Liang 93-4

Zhao Ziyang 104-5, 107, 109 